\title{
DIAGNOSTIC ACCURACY OF URINE DIPSTICK IN DETECTION OF PATIENTS OF UTI KEEPING URINE CULTURE AS A GOLD STANDARD.
}

\footnotetext{
1. MBBS, FCPS (Pediatric Medicine) Assistant Professor

Pediatric Medicine

Mayo Hospital, Lahore.

2. MBBS, PGPN (Boston University, USA)

Medical Officer Pediatrics

Govt General Hospital, Faisalabad.

3. MBBS

Faisalabad Medical University,

Faisalabad.

4. MBBS, MCPS,

FCPS (Pediatric Medicine) Associate Professor Pediatrics Allied Hospital, Faisalabad.

5. MBBS, FCPS (Pediatric Medicine) Assistant Professor Pediatric Emergency Mayo Hospital, Lahore.

6. MBBS, MD

Medical Officer

Ashraf Naseer Medical Center, Multan

7. MBBS, FCPS (Medicine)

Assistant Professor

Madinah Teaching Hospital,

Faisalabad
}

\section{Correspondence Address:}

Dr. Arslan Ahmad

Faisalabad Medical University,

Faisalabad.

ahmadarslan11@gmail.com

Article received on:

25/09/2019

Accepted for publication:

21/12/2019

\section{INTRODUCTION}

The presence of Bacteria within the proximity of symptoms in urine can be characterized as UTI. ${ }^{1}$; around $10 \%$ of girls and $30 \%$ of boys will have had a UTI by 16 years of age. The symptoms can be classical symptoms such as dysuria, urgency and abdominal pain. ${ }^{2}$ The prevalence of UTIs ranges from 1-73\% worldwide. In Pakistan UTI is one of the common problems for hospital visits in pediatric ages and girls are the most affected. ${ }^{3} \mathrm{~A}$ recent study from Karachi reported $18 \%$ prevalence of UTI in pediatric population. ${ }^{5}$

Clinical highlights recommend UTI incorporate a background history of earlier UTI, temperature
Malik Abid Ali', Muhammad Ahsan², Arslan Ahmad ${ }^{3}$, Muhammad Shamaoon ${ }^{4}$, Tehmina Maqbool ${ }^{5}$, Saad Javaid ${ }^{6}$, Salman Azhar

RACT... For children below age of five urinary tract infection (UTI) is the foremost needed as substitute to culture. Objectives: To determine the diagnostic accuracy of urine dipstick in detection of UTI in children keeping urine culture as a gold standard. Study Design: 作 experience working in the Outpatient Department, Madinah Teaching Hospital, Faisalabad. ef UTI in children on urine culture as a gold standard was recorded in $20.69 \%(n=84)$ specificity, positive predictive value, negative predictive value and accuracy rate. Conclusion: Key words: $\quad$ Children, Diagnostic Accuracy, Urinary Tract Infection, Urine Dipstick.

Article Citation: Ali MA, Ahsan M, Ahmad A, Shamaoon M, Maqbool T, Javaid S, Azhar S. Diagnostic accuracy of urine dipstick in detection of patients of UTI keeping urine culture as a gold standard. Professional Med J 2020; 27(7):14281432. DOI: 10.29309/TPMJ/2020.27.07.4195

more noteworthy than $39^{\circ} \mathrm{C}$ or $40^{\circ} \mathrm{C}$, duration of temperature for over 24 hours, suprapubic tenderness, and absence of circumcision. ${ }^{3,4}$ Moreover, the likelihood of a UTI decreases by just a little degree in the proximity of another source of fever. Abdominal ache, back ache, dysuria, recurrence and incontinence are present in older children due to UTI. nonetheless, because of the absence of reliable clinical features any child with fever without a restricting source ought to experience workup for a UTI. In children it is always preferable that these infections are diagnosed early and managed accordingly.

Urine culture is the gold standard for diagnosing 
UTIs $^{3}$, however, the time span for results is a serious issue. There are other diagnostic options available and a urine dipstick for a lab analysis may be less time consuming. The urine analysis is the most commonly used test for evaluating UTI, but it is time consuming. The diagnostic precision of dipsticks for UTIs has been studied; these examinations have demonstrated that analytic exactness of rapid dipstick tests is questionable. These studies demonstrate that dipsticks can improve indicative exactness yet ineffectively precludes infection. ${ }^{5}$

A recent study done in 2010 has shown that urine dipsticks are moderately sensitive (75\%) and less specific (66\%) in predicting UTI. ${ }^{5}$ In 2007 , American Academy of Pediatrics has discouraged the use of dipstick in detecting UTI as it has low diagnostic yield. ${ }^{6}$

In a recent randomized controlled trial done in 2010, it was concluded that starting antibiotics after the early detection of UTI is the most cost effective strategy but there are insufficient local data available. ${ }^{7}$

The aim of my study was to detect UTI early by the use of urine dipstick as it is an easy modality, an inexpensive test and we can start early treatment with antibiotics and hence can decrease the complications associated with late detection of UTI. It is not routinely performed in our hospital setting and I want to do it as an effective modality that would guide us towards the early treatment and hence prevention of complications.

\section{MATERIAL AND METHODS}

This cross sectional validation research was conducted at Outpatient department, Madinah teaching hospital, Faisalabad. The purpose of research was to decide the demonstrative precision of urine dipstick in detection of UTI in children keeping urine culture as a gold standard. Operational definitions used in this study were: Urine Dipstick: A urine test strip or dipstick was detected qualitatively whether leucocyte esterase or nitrate is present or not. Urine Culture: Growth of gram negative organism as tiny pink glistening colonies on CLED medium within 24 hour on a midstream clean catch sample or suprapubic catheterization. DIAGNOSTIC ACCURACY: Sensitivity: measures the ability of a urine dipstick to detect UTI when UTI is present. Specificity: measures the capability of a urine dipstick to correctly exclude UTI when UTI is nonexistent. True Positive: Urine dipstick test result is one that detects the condition when UTI is present also in culture. False Negative: Urine dipstick test result is one that does not detect UTI when the condition is present in urine culture report. True Negative: Urine dipstick test result is one that does not detect UTI when the condition is absent IN culture also. False Positive: Urine dipstick test result is one that detects UTI when the UTI is absent in urine culture. Positive Predictive value: is the proportion of positives that correspond to the presence of UTI by urine dipstick. Negative Predictive value: is the proportion of negative that correspond to the absence of UTI by urine dipstick. Duration of study was 6 months after approval of synopsis from: 01-2018 to 07-2018. Sample size of 406 was calculated by using sensitivity and specificity sample size calculator with following statistical assumptions: Confidence level: 95\%, Sensitivity: $75 \%{ }^{5}$ Specificity: $66 \%{ }^{5}$ Prevalence of UTI in our population $18 \%^{5}$ Sample technique used was non-probability consecutive sampling.

All females' children of age from 2 months to 5 years with fever upto $101^{\circ} \mathrm{F}$ with presenting complaints (lower /suprapubic/crampy abdominal pain), burning micturition and frequency of micturition ten to fifteen time per day were included within the research. All Patients who have taken antibiotics in the earlier 48 hours.; those with underlying congenital renal anomalies i.e. cystic kidney, obstruction in renal tract, and those with Renal Tumors, Renal Syndromes, Acute Renal failure and CKD were excluded from the study.

Every female patient age 2 months to 5 years present in the inpatient or outpatient division with signs and manifestations of urinary tract infection were incorporated after approval from the hospital ethical committee. Urine was collected using a midstream clean catch sample or supra pubic aspiration by staff nurse. 
A rapid dipstick analysis for leukocyte esterase and nitrite was done by a staff nurse using Meditest Combi 10 SGL. The reaction of dipstick strip was read visually by a trained nurse who has five year experience working in the Outpatient department, Madinah teaching hospital, Faisalabad. All dipstick positive and dipstick negative samples underwent cultures by sending samples in a clean container to the pathology lab via a sanitary attendant within one hour of the urine collection. In the lab, the urine sample was incubated in a CLED medium at $37^{\circ} \mathrm{C}$. Next day after 24 hour the lab assistance was visualized the medium to look for the growth of pink glistening colonies which was indicative of the gram negative organisms. A study proformawas used to record the information regarding patient age, gender and the results of urine dipstick and urine culture tests.

SPSS version 10 was used for the analysis. Mean and standard deviation was calculated for all quantitative variables i.e. age. By keeping urine culture as gold standard, frequency and percentage was presented for all qualitative variables i.e., true positive, false positive, false negative and true negative. Sensitivity, specificity, positive and negative predictive value was calculated using $2 \times 2$ table as follows,

\begin{tabular}{|c|c|c|c|}
\hline \multirow{3}{*}{$\begin{array}{c}\text { Urine } \\
\text { Dipstick }\end{array}$} & Test Result & Positive & Negative \\
\cline { 2 - 4 } & Positive & TP & FP \\
\cline { 2 - 4 } & Negative & FN & TN \\
\hline
\end{tabular}

Sensitivity $=\mathrm{TP} / \mathrm{TP}+\mathrm{FN}{ }^{*} 100$

Specificity $=\mathrm{TN} / \mathrm{TN}+\mathrm{FP}{ }^{\star} 100$

Positive Predictive Value $=\mathrm{TP} / \mathrm{TP}+\mathrm{FP} * 100$

Negative Predictive Value $=\mathrm{TN} / \mathrm{TN}+\mathrm{FN} * 100$

Diagnostic accuracy: TP + TN/TP + TN +FP +FN *100

\section{RESULTS}

To decide the diagnostic precision of urine dipstick in discovery of UTI in children by keeping urine culture as gold standard an aggregate of 406 cases satisfying the inclusion/exclusion criteria were selected.

Patients were distributed according to age of the patients, it shows that $69.21 \%(n=281)$ were between 2 months to 3 years of age while $30.79 \%(n=125)$ were between $4-5$ years of age, mean + sd was calculated as $2.63+1.36$ years. (Table-I)

Frequency of UTI in children on urine culture as a gold standard was recorded in $20.69 \%(n=84)$ while $79.31 \%(n=322)$ were not positive with the morbidity. (Table-II)

Diagnostic accuracy of urine dipstick in detection of UTI in children keeping urine culture as a gold standard was recorded as $80.95 \%, 62.42 \%$, $35.98 \%$, $92.63 \%$ and $66.26 \%$ for sensitivity, specificity, positive predictive value, negative predictive value and accuracy rate. (Table-III)

\begin{tabular}{|c|c|c|}
\hline Age (in years) & No. of Patients & $\%$ \\
\hline $2 \mathrm{~m}-3$ years & 281 & 69.21 \\
\hline $4-5$ years & 125 & 30.79 \\
\hline Total & 406 & 100 \\
\hline Mean+SD & \multicolumn{2}{|c|}{$2.63+1.36$} \\
\hline \multicolumn{3}{|c|}{ Table-I. Age distribution $(n=406)$} \\
\hline UTI & No. of Patients & $\%$ \\
\hline Yes & 84 & 20.69 \\
\hline No & 322 & 79.31 \\
\hline Total & 406 & 100 \\
\hline
\end{tabular}

Table-II. Frequency of UTI in children on urine culture as a gold standard $(n=406)$

\begin{tabular}{|c|c|c|c|}
\hline \multirow{2}{*}{$\begin{array}{l}\text { Urine } \\
\text { Dipstick }\end{array}$} & \multicolumn{2}{|c|}{ Urine Culture } & \multirow[b]{2}{*}{ Total } \\
\hline & $\begin{array}{c}\text { Malignant } \\
\text { (Positive) }\end{array}$ & $\begin{array}{l}\text { Malignant } \\
\text { (Negative) }\end{array}$ & \\
\hline Positive & $\begin{array}{c}\text { True } \\
\text { positive(a) } \\
68(16.75 \%)\end{array}$ & $\begin{array}{c}\text { False } \\
\text { positive (b) } \\
121(29.80 \%)\end{array}$ & $\begin{array}{c}a+b \\
189(46.55 \%)\end{array}$ \\
\hline Negative & $\begin{array}{c}\text { False } \\
\text { negative(c) } \\
16(3.94 \%)\end{array}$ & $\begin{array}{c}\text { True } \\
\text { negative(d) } \\
201(49.51 \%)\end{array}$ & $\begin{array}{c}c+d \\
217(53.45 \%)\end{array}$ \\
\hline Total & $\begin{array}{c}a+c \\
84(20.69 \%)\end{array}$ & $\begin{array}{c}b+d \\
322(79.31 \%)\end{array}$ & $406(100 \%)$ \\
\hline
\end{tabular}

Table-III. Diagnostic accuracy of urine dipstick in detection of UTI in children keeping urine culture as a gold standard $(n=406)$

Sensitivity $\quad=a /(a+c) \times 100=80.95 \%$

Specificity $=d /(d+b) \times 100=62.42 \%$ 
Positive predictive value $=\mathrm{a} /(\mathrm{a}+\mathrm{b}) \times 100=35.98 \%$

Negative predictive value $=d /(d+c) \times 100=92.63 \%$

Accuracy rate $=a+d /(a+d+b+c) \times 100=66.26 \%$

\section{DISCUSSION}

For children below age of five urinary tract infection (UTI) is the foremost common sources of infection. To lessen the danger of renal scarring it is critical to have immediate diagnosis and treatment. Quick, cost-effective, methods for the analysis of UTI are needed as substitute to culture.

The current study was planned to detect UTI early by the use of urine dipstick as it is an easy modality, an inexpensive test and we can start early treatment with antibiotics and hence can decrease the complications associated with late detection of UTI. It is not routinely performed in our hospital setting so we wanted to do it as an effective modality that may guide us towards the early treatment and hence prevention of complications.

In our study, $69.21 \%(\mathrm{n}=281)$ were between 2 months to 3 years of age while $30.79 \%(n=125)$ were between 4-5 years of age, mean+sd was calculated as $2.63+1.36$ years, frequency of UTI in children on urine culture as a gold standard was recorded in $20.69 \%(n=84)$, the diagnostic accuracy of urine dipstick in detection of UTI in children keeping urine culture as a gold standard was recorded as $80.95 \%, 62.42 \%, 35.98 \%$, $92.63 \%$ and $66.26 \%$ for sensitivity, specificity, positive predictive value, negative predictive value and accuracy rate.

A recent study done in 2010 has shown that urine dipsticks are moderately sensitive (75\%) and less specific (66\%) in predicting $\mathrm{UTI}^{5}$, our study findings are in agreement with the above study, contrary to above, in 2007, American Academy of Pediatrics has discouraged the use of dipstick in detecting UTI as it has low diagnostic yield. ${ }^{6}$

In a recent randomized controlled trial done in 2010, it was concluded that starting antibiotics after the early detection of UTI is the most cost effective strategy but there are insufficient local data available. ${ }^{7}$

Smith $\mathrm{P}$ and others $^{8}$ evaluated the sensitivity, specificity, positive and negative predictive values of Negative microscopy and negative dipstick to predict culture results: absence of a reportable pathogen and recorded sensitivity $83 \%$, specificity $76 \%$, PPV $94 \%$, NPV $76 \%$, which is supporting to the current study.

Another study 9 included paper for children (aged 3 weeks-21 years) with suspected UTI a n d recorded that sensitivity $92 \%$, specificity $62 \%$, PPV 22\%, NPV 99\%. Microscopy: Sensitivity 92\%, specificity 49\%, PPV 17\%, NPV 98\%.

The latest research done by Whiting et $\mathrm{al}^{10}$ exploring the diagnostic accurateness of dipstick analysis and microscopy for UTI in kids is an all around directed efficient review. The overall conclusion is that negative dipstick analysis for leukocyte esterase and nitrite, or microscopy negative for bacteriuria and pyuria can be used to rule out UTI, without the need for confirmatory culture. Positive dipstick examination and microscopy can likewise be utilized to prompt further examination, by sending for urine culture to affirm UTI. To clinical practice the value of these conclusions is that use of such quick diagnostic tests would result in saving clinic charges by not having to send for culture in case tests are negative, conjointly result in less children getting improper antibiotic treatment whereas anticipating tests results of culture.

In summary, the results of our study support the use of urine dipstick to detect UTI early as it is an easy modality, an inexpensive test and we can start early treatment with antibiotics and hence can decrease the complications associated with late detection of UTI. Though, it is not routinely performed in our hospital setting however, considering the outcome of our research in accordance with other studies, the use of this effective modality may guide us towards the early treatment and hence prevention of complications.

\section{CONCLUSION}

We conclude that the analytic precision of urine 
dipstick in detection of UTI in children keeping urine culture as a gold standard is higher and this cost effective and easy technique may be used in our routine practice for prediction of UTI in children.

\section{Copyright $\odot 21$ Dec, 2019.}

\section{REFERENCES}

1. Hutchings F, JadresicL. GPs should evaluate all children following UTI. Practitioner 2010; 254(1731):179.

2. Falakaflaki B, Mousavinsab SN, Mazloomzadeh S: Department of pediatrics, dipstick urinanlysis screening of healthy neonates. PediatrNeonatol2011; 52(3):161-4.

3. Najeeb S, Munir T, Rehman S, Hafiz S, Hafiz A, Gilani $A$ et al. Comparison of urine dipstick test with conventional urine culture in diagnosis of urinary tract infection. J Coll Physicians and Surg Pak 2015; 25(2):108-10.

4. Ali SR, Ahmed S, Nizami s, Qadir M. Do all pediatric urine specimens need to go to the laboratory? Int J Child Health Nutr 2012; 1:66-71.
5. Little P, Turner S, Rumsby K, Jones R, Warner G, Moore $M$. Validating the prediction of lower urinary tract infection in primary care: Sensitivity and specificity of urinary dipstick and clinical scores in women. $\mathrm{Br} J$ Gen Pract 2010; 60(576):495-500.

6. Sekhar DL, Wang L, Christopher S, Hollenbeak M, Widome. Ian MP. A cost effectiveness analysis of screening urine dipsticks in well-child care. Pediatrics 2010; 125(4):660-3.

7. Turner D, Little P, Raftery J, Turner S, Smith H, Rumsby $t$ et al. Cost effectiveness of management strategies for urinary tract infections: Results from Randomized Controlled Trial: BMJ 2010; 340:c346.

8. Smith P, Morris A, Reller LB. Predicting urine culture results by dipstick testing and phase contrast microscopy. Pathology 2003; 2:161-5.

9. American Academy of Pediatrics. Committee on Quality Improvement. Practice parameter: The diagnosis, treatment, and evaluation of the initial urinary tract infection in febrile infants and young children. Pediatrics 1999; 103(4 Pt 1):843-52.

10. Penny Whiting, Marie Westwood, Ian Watt, Julie Cooper, and Jos Kleijnen Rapid tests and urine sampling techniques for the diagnosis of urinary tract infection (UTI) in children under five years: A systematic review [pre-publication paper ONLINE accessed 14/06/05]. Biomed Central Paediatrics 2005; 5:4.

\begin{tabular}{|c|l|l|l|}
\hline \multicolumn{2}{|c}{ AUTHORSHIP AND CONTRIBUTION DECLARATION } \\
\hline Sr. \# & \multicolumn{1}{|c|}{ Author(s) Full Name } & \multicolumn{1}{|c|}{ Contribution to the paper } & Author(s) Signature \\
\hline 1 & Malik Abid Ali & Data entry, Paper writing. & \\
\hline 2 & Muhammad Ahsan & $\begin{array}{l}\text { Data analysis, Paper writing, } \\
\text { Data collection. } \\
\text { Data entry, Result writing, Data } \\
\text { collection. }\end{array}$ \\
\hline 5 & Arslan Ahmad & $\begin{array}{l}\text { Muhammad Shamaoon } \\
\text { Data analysis, Discussion } \\
\text { writing, Data collection. } \\
\text { Data collection, Data entry, }\end{array}$ \\
\hline 6 & Tehmina Maqbool & $\begin{array}{l}\text { Sata analysis. } \\
\text { Data entry, paper writing. }\end{array}$ \\
\hline 7 & Salman Azhar & Data collection, Data analysis. \\
\hline
\end{tabular}

